\title{
LOS DERECHOS HUMANOS EN ECUADOR: UNA VISIÓN PESIMISTA*
}

\author{
Fredy Rivera Vélez **
}

La reivindicación de varios derechos colectivos e individuales en el Ecuador, por lo general, ha estado vinculada a la forma en que se ha pensado y diseñado la política y la sociedad, y más precisamente, en cómo los regímenes y sus pobladores han asumido en términos gruesos lo que se entiende por derechos.

Para nadie es desconocido que nuestro país es carente de una serie de situaciones sociales y económicas que garanticen la vida con dignidad. Tampoco produce asombro la persistencia del racismo como pauta que marca las relaciones cotidianas de la gente. De igual forma, la sociedad en conjunto no parece inmutarse ante la legitimación de la exclusión social como política pública normalmente aceptada. Es más, se suele mencionar que eso es lo políticamente correcto desde una perspectiva coherente e instrumental para quienes nos diseñan el convivir en todas las dimensiones de la vida. Al final, los costos sociales de los largos ajustes que vivimos desde los años 80 y sus efectos, han calado tan profundamente en nosotros que nos hemos adaptado pacientemente a esas contingencias.

Es común también aceptar el hecho de que, como reza el refrán popular, "así mismo somos" lleva implícito una aceptación cuasi natural de las desigualdades e injusticias en las que nos vemos insertos. Pero esta aceptación, que parecería más un lamento, representa en el fondo la presencia y acción de una serie de perversidades.

La primera está relacionada con la tan trillada idea de la ciudadanía plena.

Definitivamente, la construcción de ciudadanía total en el país es un proceso en ciernes, o mejor dicho, es una buena muestra de lo que somos capaces de hacer en términos de plasmar imaginarios sociales. En este punto basta con preguntarnos desde cuándo votan los analfabetos (buena parte indígenas) en este país, o simplemente, desde cuándo se despenalizaron los homosexualismos, o en qué año, a la mujer se le permitió denunciar a sus agresores familiares. La respuesta es simple: hace poco. Y eso, solamente para citar algunos ejemplos relacionados con el ámbito del sufragio, con la situación de las minorías y con el mundo de la familia.

Desde ese punto de vista, la vieja idea de la democracia liberal, basada en la homogenización del derecho para todos, un derecho que sea extendido sin discriminación, viene a representar una especie de abstracción o esquizofrenia colectiva si la pensamos desde el real divorcio existente entre la cantidad inimaginable de leyes que se han creado en diversas etapas de la historia política ecuatoriana y las prácticas concretas de su aplicación sobre la población. Para muestra un botón: la profunda corrupción existente en el sistema de administración de justicia y el descrédito de los organismos de control y seguridad pública.

Pero el déficit del derecho ciudadano no es solamente culpa del Estado nacional. Con esta afirmación no quiero dejar limpia la imagen y responsabilidad de este y de otros gobiernos de turno que han fracasado en lo que se ha denominado "paquete civilizador". Simplemente deseo recalcar que la internalización de la idea y práctica ciudadana, también tiene una doble vía de entrada desde las personas, la gente.

No es cuestión de que se desarrollen una serie de programas educativos que prioricen la cultura cívica y formación ciudadana, que sí son importantes en un momento determinado. El telón de fondo es que en Ecuador, esos diversos programas e intencionalidades, sean éstos venidos de Ongs, Estado, partidos y otras entidades, no han dimensionado correctamente las matrices de donde provienen las aceptaciones cuasi naturales del déficit de derechos y de los órdenes excluyentes establecidos.

Un ejemplo, en Ecuador, las relaciones sociales siempre fueron determinadas por dependencias personales. En forma reducida y parcial se han abolido estas relaciones personales en el siglo XX, sobre todo en las ciudades y de manera restringida en varios ámbitos rurales a pesar de reformas agrarias, proyectos de desarrollo rural integral, trabajo eclesial de base, esfuerzos de Ongs de todo color y todos los inventos de esa naturaleza.

Del buen o mal patrón pasamos al clientelismo o personalismo político y cultural.

* Una versión de este artículo fue presentado en el Segundo Foro Nacional "Construyendo una agenda nacional para la promoción de los Derechos económicos, sociales y culturales” realizado en Flacso Ecuador en junio de 1998.

** Coordinador del Diploma de Derechos Humanos y Seguridad Democrática de FLACSO Ecuador. Editor de la Revista ECUADOR DEBATE del Centro Andino de Acción Popular (CAAP). 
No solamente se trata de regímenes políticos basados fuertemente en el clientelismo, sino de un rasgo cultural que hasta la fecha marca las relaciones sociales en el Ecuador y que dice mucho del déficit de la construcción de las instituciones políticas y de la democracia en el país ${ }^{1}$.

Este tipo de situaciones se entienden mejor con casos y anécdotas. Una de ellas contada precisamente por gente sencilla y que me servirá de ejemplo para sustentar lo antedicho.

"Un hombre pobre, que trabajaba durante años como chofer en una empresa de transporte en el sector de la construcción, contó que, cuando él habia logrado aborrar, y unos amigos le habian prestado otra parte del dinero para comprar su propia camioneta, su jefe le reprochó por no baber acudido a él para el préstamo. Según el chofer, su ensayo de independizarse un poco no fue apreciado por su jefe. Este jefe no pudo impedir que su trabajador comprara algo para complementar su ingreso en los sábados libres. Pero no le gustó la idea que su empleado no acudió a él para el préstamo. Comentó el chofer: «es que cuando uno trabaja en alguna parte, tus jefes «siempre quieren tenerte el pie sobre el cuello para que dependas de ellos... alli te pueden exigir que trabajes basta las 8 de la noche...»Y él se sintió feliz de no baber tenido la obligación de pedir prestado plata donde su jefe, porque ello implicaba reproducir una situación tradicional de subordinación real y simbólica" (Salman, 1998: 10).

Este patrón de dependencias personales marca muy fuerte las relaciones sociales en Ecuador hasta hoy día. Para obtener un préstamo, para que tu hijo tenga acceso a alguna escuela o universidad, para que te arreglen algún asunto en la municipalidad, te sirven -solamente- tus palancas, amigos, intermediarios y muchas veces, incluso, puedes tratar de movilizar a tus superiores en el trabajo.

Como consecuencia, las instituciones son inmóviles, e inconcebibles y herméticas. Y la institucionalidad política es un mundo que va más allá del poder de influencia de uno. La percepción de «los circuitos de toma de decisiones son opacos, misteriosos, existen redes escondidas, que usan lenguajes excluyentes» ${ }^{2}$. Qué decir de mi derecho ciudadano a pedir y exigir rendición de cuentas, peor aún a que me vengan con el cuento de la democracia participativa, por más esfuerzos que hagan los diseñadores políticos, creyentes sumisos de la nueva moda del "empoderamiento", de la real politik y la descentralización efectiva.

$Y$ es que estas situaciones contribuyen para generar un ambiente legitimador de una ciudadanía llena de peripecias, donde solamente las estrategias de afirmar la autoridad y dignidad de los poderosos sirve para obtener bienes, ni hablar de derechos.

No se percibe la institucionalidad política, ni el espacio público como transparente, o fundado en procedimientos unívocos. Es un mundo donde uno solamente tiene acceso por amistades, y si éstas faltan, por suplicaciones, como en tiempos del patrón, sino que ahora se llaman autoridades gubernamentales, redes partidarias, instituciones de distinto signo. Es decir, el peso de esa fuerte "comunalización mental", de esa condición de longos ${ }^{3}$, a decir de recientes estudios, todavía subsiste fuertemente en nuestra matriz socio-política.
Siempre fue, y aún está presente en la cultura ecuatoriana, el autoritarismo personal, el respeto para la autoridad en lo público y en lo privado. Nunca se democratizó el poder del individuo que, con su esfuerzo, su astucia, su inteligencia, pueda decidir sobre su suerte y la suerte de su familia. Las estructuras de exclusión, de desigualdad y de privilegios, siempre fueron mucho más fuertes que el ánimo del individuo. Esa especie de actor racional, basado en una cultura ciudadana, portador de derechos otorgados o por ser otorgados, simplemente es inexistente o se limita a determinados segmentos poblacionales.

Si partimos del hecho de que, teóricamente, la ciudadanía no es la suma de individuos, sino que implica la existencia de pautas culturales que tengan en su base el reconocimiento del otro como igual, tenemos la segunda situación perversa de que nuestra gente no se reconoce como igual; es más, sustenta, expande y legitima esa desigualdad por considerarla cuasi natural "así mismo es".

No quiero entrar en explicaciones obvias sobre la importancia de los procesos de dominación y poder que han estado presentes en ese tipo de construcciones sociales. Simplemente quiero dejar en claro que la persistencia de esas matrices culturales que orientan la vida en común de la "ciudadanía" es un dato duro con el que debemos trabajar. Por eso se pone hincapié en el asunto de las culturas políticas, y conste que no digo cultura política. El solo hecho de mencionar la acepción es una aceptación concreta de nuestra diversidad.

Entender lo anterior implica reconocer que en nuestro medio tiene menos peso la ley, la regla, el procedimiento claro y el conocimiento sobre derechos y normas. Nos caracterizamos por tener desconfianza en la imparcialidad y profesionalidad de funcionarios públicos, con toda razón; somos reyes para maniobrar en circunstancias caprichosas, pero vasallos para el reclamo de derechos, para exigir transparencia, pedir igualdad y recibir lo merecido, es decir, ciudadanos de una sola entrada.

El resultado de todo ello es una amplia gama de estrategias, trucos y mucho espacio para mantener de tradiciones propias, rasgos particulares, e idiosincrasias grupales. También el resultado es mucha tolerancia para dejar de lado la propia dignidad personal, lo cual constituye, o podría constituir, una estrategia para lograr cosas, porque

1. Al respecto se han realizado muchas investigaciones. Las recientes abordan precisamente temas relacionados con los ámbitos de la cultura política. Al respecto ver: BURBANO DE LARA, Felipe: "Cultura política y democracia en el Ecuador" Documento de trabajo N. 10, CORDES, Konrad Adenaüer Fundation, Cooperación Española, Quito, septiembre de 1998.

2. SALMAN, Tom: "Culturas políticas e identidades populares urbanas: los casos de Chile y Ecuador" en: Ecuador Debate N. 44, CAAP, Quito, 1998.

3. "Longos" es una figura y categoría de ubicación social, utilizada preferentemente por la población blanco mestiza del país, fuertemente cargada de estigmas y connotaciones racistas, que hace relación a una condición de identificación de lo popular con lo étnico (indígena). La utilización de esta figura en las prácticas cotidianas implica discriminación y exclusión. En el campo de los derechos, ser "longo" acarrea la imposibilidad de reivindicar ciudadanías plenas aunque lo "legal" aparezca como una instancia que invisibiliza tales prácticas que de hecho se producen en el medio. 
subraya el poder real o simbólico del otro (el supuestamente igual, desde el punto de vista ciudadano).

Las condensaciones o figuras discursivas "no sea malito", "haga el favorcito", no son simplemente entronques comunicativos; expresan y dicen mucho acerca del porqué la tradición influye grandemente en cómo funcione la noción de ciudadanía en el país.

Aparte del problema general de que ciudadanía no es una cosa que se puede decretar, o que no es una cosa que se realice «automáticamente», pareciera que en el país los pobladores, esos sujetos que tendrían la tarea de reivindicar sus derechos, muchas veces rechazan los valores detrás del concepto clásico de ciudadanía como individuo, como portador de derechos, autonomía y soberanía individual, es decir, mantienen una actitud ambigua.

Si eso es así, o por lo menos lo planteamos hipotéticamente de esa forma, entonces el actual debate en torno a la ciudadanía y la construcción de diversa índole de derechos, y todos los esfuerzos y proyectos que existen para estimular, promover y concretizar la ciudadanía, se ven confrontados con una «inconmensurabilidad» mucho más allá de la enseñanza, la explicación, o la simple implementación. En Ecuador, por ejemplo, en los conceptos y en la cotidianidad de los pobres, en su mundo de vida, en el cómo salir adelante con su familia, y cómo sobrevivir y «arreglarse» el diario, no cabe así no más la idea de ciudadanía y de derechos como se los ha procesado en sociedades industriales y postindustriales.

Es cuestión de observar comportamientos. La gente pobre trata de evitar los enfrentamientos «no personales» entre la ley y sus representantes y ellos mismos, porque saben que solamente, relaciones personales pueden cambiar el supuesto NO del ejecutor de la ley en un SI para ellos. O sea: toda la vida es una enseñanza hacia la no-factibilidad de la igualdad ante la ley, y de lo insensato de insistir en sus derechos y su dignidad como individuo. En ese sentido, la internalización de todas estas experiencias, y de las estrategias no ciudadanas de «la gente» que no puede hacerse valer como portadora de «derechos», produce que el discurso clásico sobre ciudadanía se reciba con escepticismo.

Por eso existen situaciones ambiguas donde la ciudadanía funciona, forma parte de una imagen y está presente como mecanismo para obtener cosas, para ser atendido, por lo menos para ejecutar determinados intereses, lo cual nos acercaría a una concepción instrumental y conscientemente utilitaria de la funcionalidad de la ciudadanía y los derechos que ella conlleva. Pero pensar que esas acciones desplegadas por las personas sean parte constitutiva de un cuerpo de derechos ciudadanos, humanos, asumidos a plenitud, y como instancia aglutinante de identidades colectivas, estaríamos hablando de otra sociedad y otra cultura.

En Ecuador la formación limitada de ciudadanía no sólo se debe al corto arraigo de una cultura e institucionalidad democrática, sino incluso a una percepción tradicional de las mismas personas que no llegan a interiorizar el juego básico derechos y obligaciones-responsabilidades. Conceptos y auto-identificaciones que son un verdadero lastre para quienes se encuentran en la noble tarea de promover derechos ciudadanos, ya que ello exige dimensiones cívicas complejas que concilien la autonomía privada del individuo con la de una sociedad regulada por los principios de justicia e igualdad.

Dicha situación se complica aún más, cuando los ciudadanos asisten pasivamente al deterioro de las capacidades del Estado para absorber las demandas poblacionales, a la desintegración de los niveles mínimos de solidaridad social, como es la indolencia generalizada frente a los desastres del fenómeno del Niño (indolencia principalmente serrana diría yo), y frente a lo que algunos investigadores mencionan como una sociedad al borde de la anomia.

Podría resultar fatalista con estas breves ideas expuestas, pero los datos de la realidad en estos últimos tiempos no me permiten ser optimista. Un gobierno interino manejado con estrechez de criterio y acorde a la estatura de quien lo presidió. Duplicidad y sobreposición de instituciones que diseñan nuestras nuevas leyes y ante quienes deberíamos demandar y exigir los derechos que hoy nos convocan; un supuesto defensor del pueblo y ciudadanos, que ni siquiera pudo ejercer virtualmente su cargo; inseguridad ciudadana que nos conduce a promover justicia por mano propia; miedo a que se esfume nuestro dinero en instituciones bancarias de excelencia y calidad total; proliferación de profesionales yuppies con dudosos y pomposos MBA criollos, obtenidos a la luz de modas profesionales; y en general, un sistema político que a mediano plazo no garantizará escenarios propicios para sostener una vida digna en términos de derechos y paz.

Ante tal magnitud de sucesos y situaciones:¿Dónde queda la labor de distintos órganos e instituciones de promoción de derechos sociales, económicos y culturales?

Quedo con la duda, pero también con la esperanza de que se pueda abrir un ducto en el agujero negro que representa hoy en día la relación ciudadanos-sociedad y Estado. Una tarea de tal dimensión bien vale la pena apoyarla con fe, ya que la racionalidad ha fracasado. 


\section{RESUMEN}

La reivindicación de los derechos humanos en Ecuador tiene que ver con la percepción generalizada que se tiene de esos derechos y de la idiosincrasia de la sociedad. Para el autor, la vulneración de los derechos humanos no sólo proviene del Estado, sino también concierne a los ciudadanos. Así, la cultura política y social del Ecuador ha estado permeada por el clientelismo, sistema que ha generado relaciones de dependencia y dominación que confirman el poder en un sector, que impiden el desarrollo de la ciudadanía y hacen imposible la democratización del poder.

La legalidad está al margen de la vida política y social, lo que permite que reglas mínimas como son los derechos humanos sean desconocidos y la sociedad no se plantee su reivindicación. La cultura política y social es fundamental para entender el escepticismo y la entrega a normas de conducta contrarias al ejercicio de la ciudadanía y en últimas a la consolidación de la dignidad.

Palabras clave: Autoritarismo personal, desigualdad, derechos humanos, Ecuador, cultura política, ciudadanía.

\section{ABSTRACT}

The reivindication of human rights in Ecuadror relates to the generalized vision of the population regarding those rights. For the author, the transgression of these rights does not only stem from the state, but also from the citizens. Social and political culture in Ecuador has been characterized by clientelism, dependence, and domination. This cultural system hampers the development of citizenship and makes democratization impossible. Social and political life is not affected by the rule of law, which prevents human rights from being widely known and demanded. Social and political culture is crucial to understand the scepticism and the prevalence of behaviors which are inimical to citizenship and human dignity.

Key words: personal authoritarianism, inequality, human rights, Ecuador, political culture, citizenship.

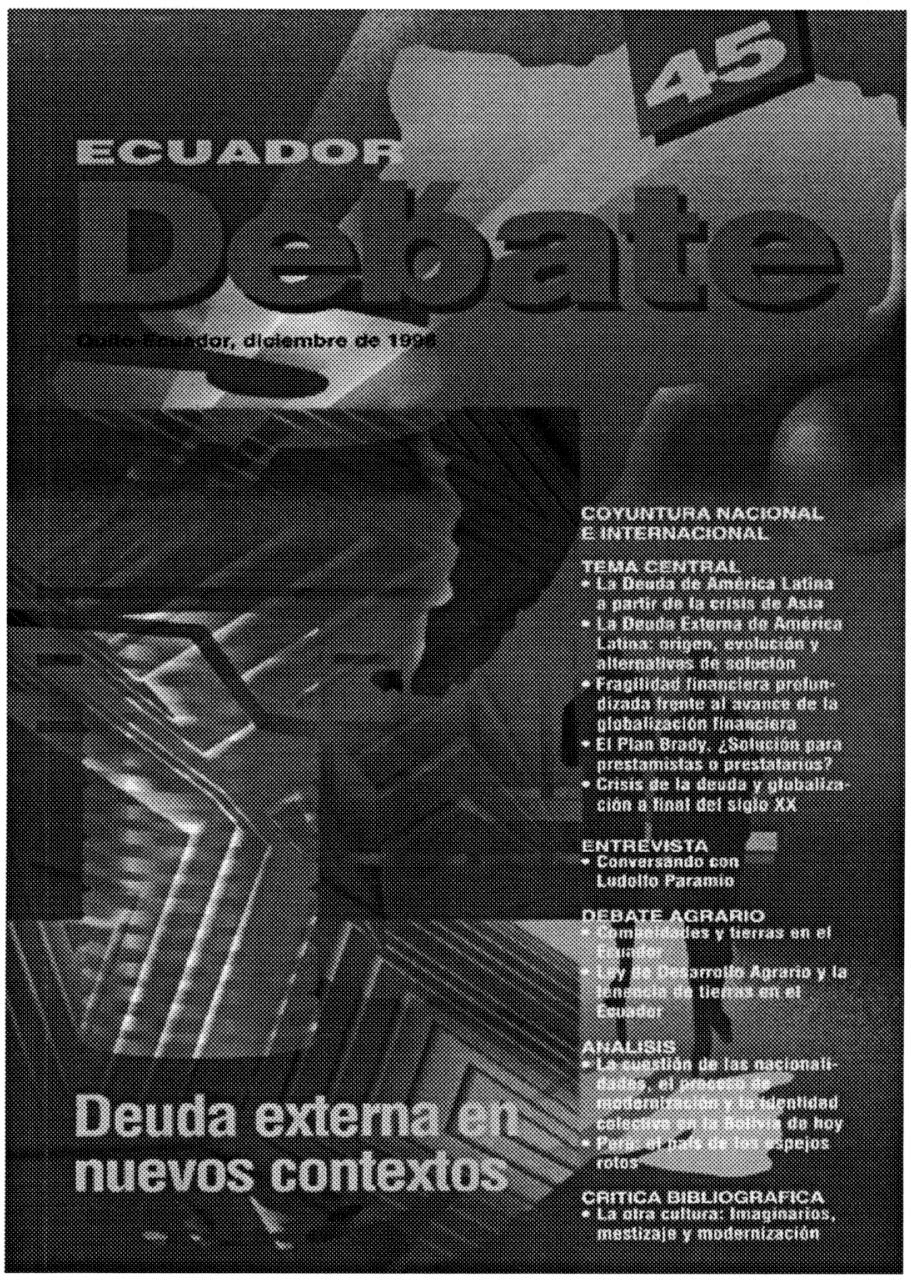

